(C) American Chemical Society, Chem. Mater., Kruk et al., cm034911u Supporting Info Page S1

\title{
Exceptionally High Stability of Copolymer-Templated Ordered Silica with Large Cage-like Mesopores
}

\author{
Michal Kruk, Ewa B. Celer and Mietek Jaroniec \\ Department of Chemistry, Kent State University, Kent, Ohio 44242
}

Supporting Table 1S. Structural properties calculated from nitrogen adsorption data for the FDU-1 sample IIa calcined at different temperatures. ${ }^{\mathrm{a}}$

\begin{tabular}{|c|c|c|c|c|c|c|c|}
\hline Sample & $\begin{array}{c}\mathrm{S}_{\text {BET }} \\
\left(\mathrm{m}^{2} \mathrm{~g}^{-1}\right)\end{array}$ & $\begin{array}{c}\mathrm{V}_{\mathrm{t}} \\
\left(\mathrm{cm}^{3} \mathrm{~g}^{-1}\right)\end{array}$ & $\begin{array}{c}\mathrm{w}(\delta) \\
(\mathrm{nm})\end{array}$ & $\begin{array}{c}\mathrm{V}_{\mathrm{mi}} \\
\left(\mathrm{cm}^{3} \mathrm{~g}^{-1}\right)\end{array}$ & $\begin{array}{c}\mathrm{S}_{\mathrm{p}} \\
\left(\mathrm{m}^{2} \mathrm{~g}^{-1}\right)\end{array}$ & $\begin{array}{c}\mathrm{V}_{\mathrm{p}} \\
\left(\mathrm{cm}^{3} \mathrm{~g}^{-1}\right)\end{array}$ & $\begin{array}{c}\mathrm{S}_{\mathrm{ex}} \\
\left(\mathrm{cm}^{3} \mathrm{~g}^{-1}\right)\end{array}$ \\
\hline IIa(-C813) & 950 & 0.77 & $11.3(2.5)$ & 0.29 & 290 & 0.44 & 20 \\
\hline IIa-C973 & 790 & 0.66 & $10.8(2.3)$ & 0.23 & 250 & 0.39 & 20 \\
\hline IIa-C1073 & 710 & 0.61 & $10.6(2.3)$ & 0.20 & 240 & 0.37 & 20 \\
\hline IIa-C1173 & 530 & 0.49 & $9.9(2.2)$ & 0.14 & 210 & 0.32 & 20 \\
\hline IIa-C1273 & 320 & 0.34 & $9.5(2.2)$ & 0.06 & 170 & 0.26 & 20 \\
\hline
\end{tabular}

${ }^{a}$ Notation: $\mathrm{S}_{\mathrm{BET}}$, BET specific surface area; $\mathrm{V}_{\mathrm{t}}$, total pore volume; $\mathrm{w}$, primary mesopore diameter (calculated using a method developed for cylindrical rather than spherical pores; the actual pore diameters are likely to be larger by about $2 \mathrm{~nm}) ; \delta$ - the width (nm) of the pore size distribution at the half of its height is given in brackets; $\mathrm{V}_{\mathrm{mi}}$, micropore volume (including the volume of the connecting pores, if the latter are of diameter below about $4 \mathrm{~nm}$ ); $\mathrm{S}_{\mathrm{p}}$, primary mesopore surface area; $V_{\mathrm{p}}$, primary mesopore volume; $\mathrm{S}_{\mathrm{ex}}$, external surface area. 
(C) American Chemical Society, Chem. Mater., Kruk et al., cm034911u Supporting Info Page S2

Supporting Table 2S. Structural properties determined from nitrogen adsorption data for the FDU-1 samples before and after surface modification with TMS and/or heating in water at $373 \mathrm{~K}$ for 4 days. ${ }^{\mathrm{a}}$

\begin{tabular}{|c|c|c|c|c|c|c|c|}
\hline Sample & $\begin{array}{c}\mathrm{S}_{\text {BET }} \\
\left(\mathrm{m}^{2} \mathrm{~g}^{-1}\right)\end{array}$ & $\begin{array}{c}\mathrm{V}_{\mathrm{t}} \\
\left(\mathrm{cm}^{3} \mathrm{~g}^{-1}\right)\end{array}$ & $\begin{array}{c}\mathrm{w}(\delta) \\
(\mathrm{nm})\end{array}$ & $\begin{array}{c}\mathrm{V}_{\mathrm{mi}} \\
\left(\mathrm{cm}^{3} \mathrm{~g}^{-1}\right)\end{array}$ & $\begin{array}{c}\mathrm{S}_{\mathrm{p}} \\
\left(\mathrm{m}^{2} \mathrm{~g}^{-1}\right)\end{array}$ & $\begin{array}{c}\mathrm{V}_{\mathrm{p}} \\
\left(\mathrm{cm}^{3} \mathrm{~g}^{-1}\right)\end{array}$ & $\begin{array}{c}\mathrm{S}_{\mathrm{ex}} \\
\left(\mathrm{cm}^{3} \mathrm{~g}^{-1}\right)\end{array}$ \\
\hline IId & 940 & 0.83 & $11.0(2.4)$ & 0.27 & 320 & 0.51 & 30 \\
\hline IId-B4d & 560 & 0.91 & $12.6(2.8)$ & 0.04 & 420 & 0.80 & 50 \\
\hline IId-TMS & 370 & 0.45 & $10.3(2.3)$ & 0.05 & 240 & 0.37 & 20 \\
\hline IId-TMS-B4d & 420 & 0.50 & $10.2(2.3)$ & 0.05 & 270 & 0.39 & 40 \\
\hline IIe & 890 & 0.77 & $10.6(2.2)$ & 0.26 & 300 & 0.47 & 30 \\
\hline IIe-B4d & 420 & 0.78 & $12.1(3.0)$ & 0.00 & 370 & 0.70 & 50 \\
\hline IIe-TMS & 430 & 0.46 & $10.0(2.2)$ & 0.09 & 220 & 0.33 & 20 \\
\hline IIe-TMS-B4d & 480 & 0.52 & $10.0(2.5)$ & 0.06 & 310 & 0.41 & 30 \\
\hline III & 920 & 0.93 & $11.7(2.4)$ & 0.18 & 480 & 0.68 & 40 \\
\hline III-B4d & 550 & 1.24 & $14.8(6.6)$ & 0.00 & 440 & 1.06 & 120 \\
\hline III-TMS & 430 & 0.50 & $11.3(2.4)$ & 0.08 & 250 & 0.39 & 20 \\
\hline III-TMS-B4d & 400 & 0.51 & $11.2(2.6)$ & 0.04 & 270 & 0.41 & 50 \\
\hline
\end{tabular}

${ }^{a}$ Notation: see Supporting Table 1. 
(c) American Chemical Society, Chem. Mater., Kruk et al., cm034911u Supporting Info Page S3

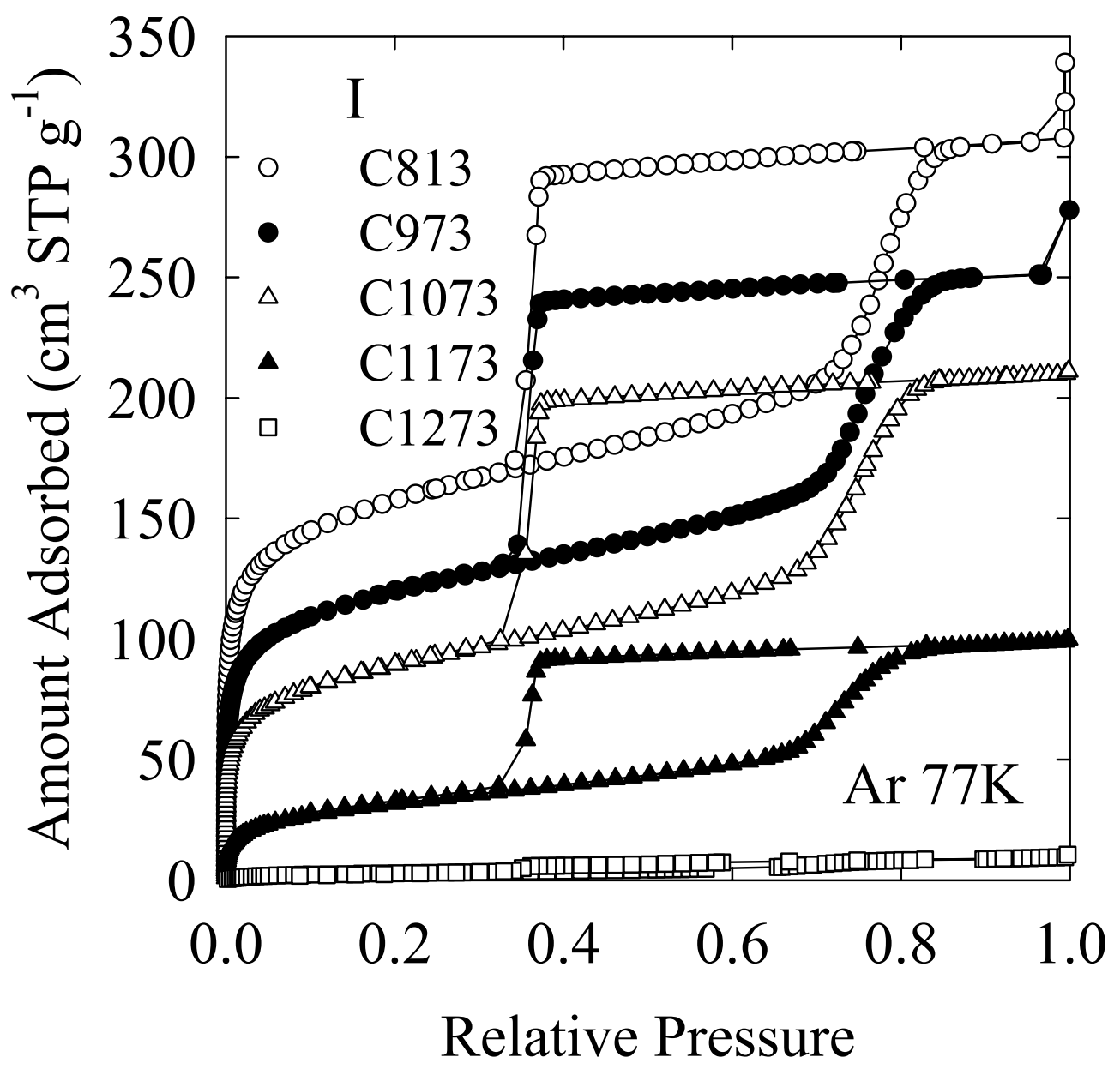

Supporting Figure 1S. Argon adsorption isotherms for FDU-1 sample I calcined at temperatures $813-1273 \mathrm{~K}$. 
(c) American Chemical Society, Chem. Mater., Kruk et al., cm034911u Supporting Info Page S4

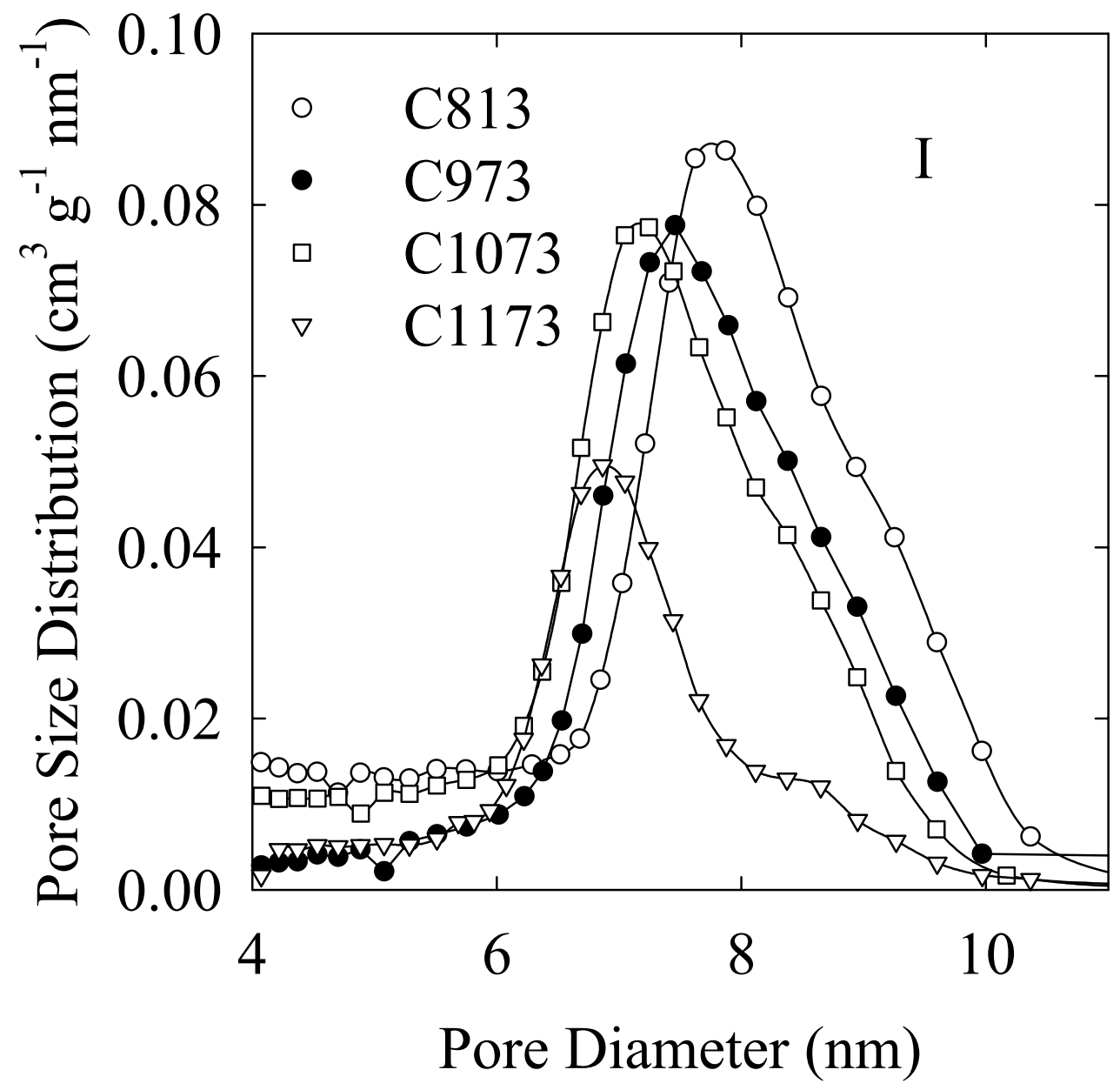

Supporting Figure 2S. Pore size distributions calculated from nitrogen adsorption isotherms for FDU-1 sample I calcined at temperatures 813-1173 K. 
(C) American Chemical Society, Chem. Mater., Kruk et al., cm034911u Supporting Info Page S5

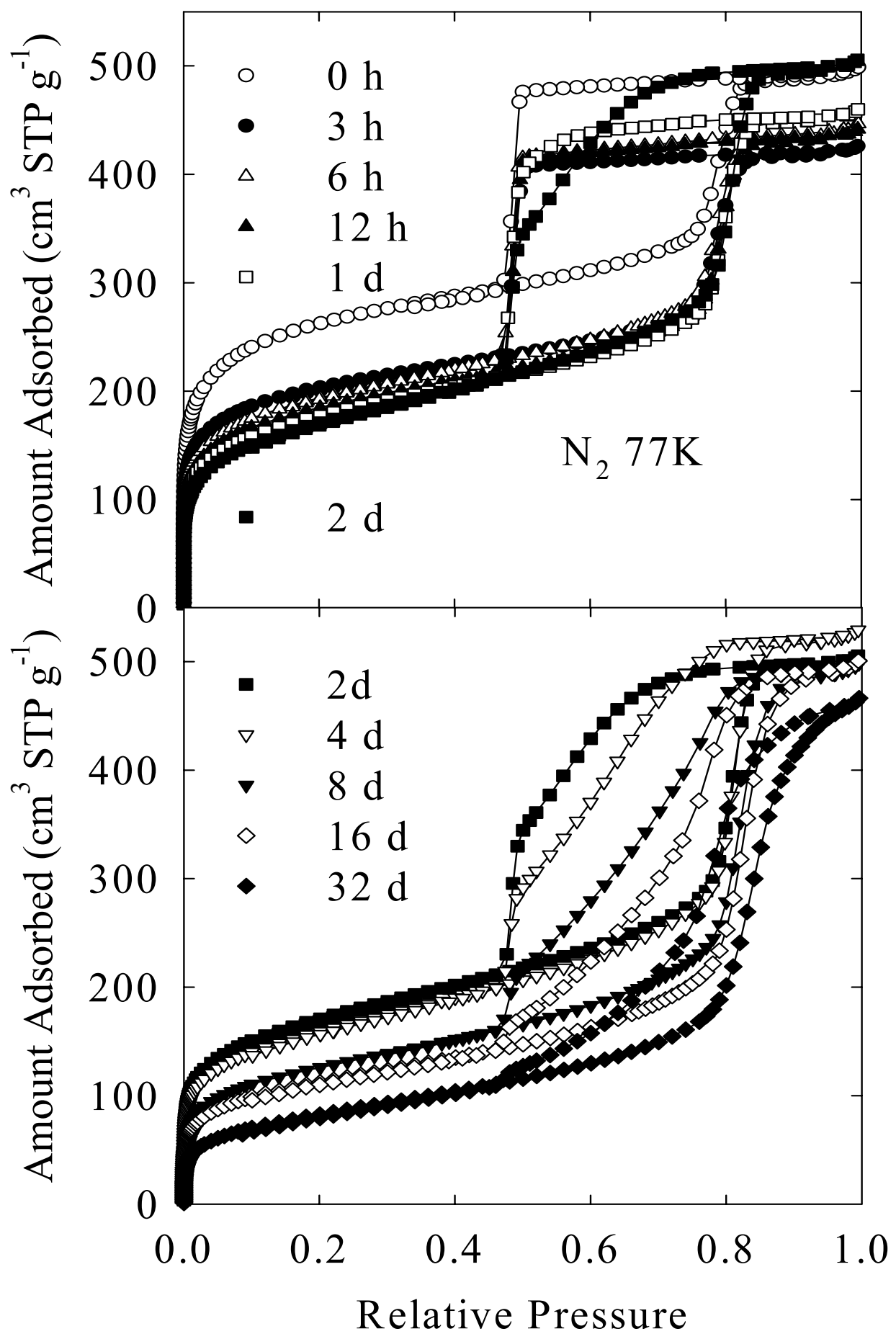

Supporting Figure 3S. Nitrogen adsorption isotherms for FDU-1 sample IIb before and after boiling for periods of time from 3 hours to 32 days. 
(c) American Chemical Society, Chem. Mater., Kruk et al., cm034911u Supporting Info Page S6

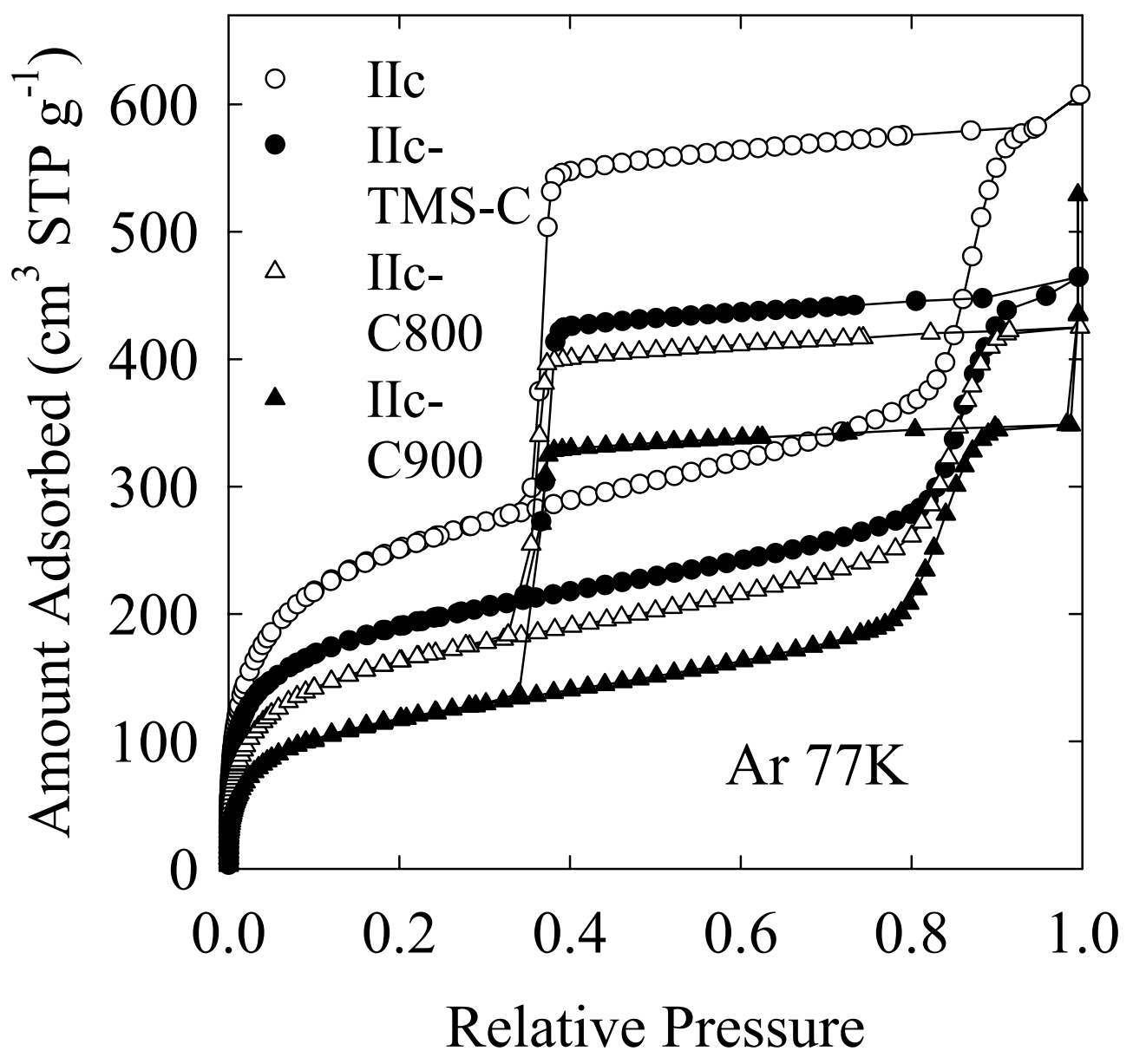

Supporting Figure 4S. Argon adsorption isotherms for FDU-1 sample IIc: calcined at $813 \mathrm{~K}$; calcined at $813 \mathrm{~K}$, modified with TMS and calcined at $813 \mathrm{~K}$; calcined at $1073 \mathrm{~K}$ and calcined at $1173 \mathrm{~K}$. 
(c) American Chemical Society, Chem. Mater., Kruk et al., cm034911u Supporting Info Page S7

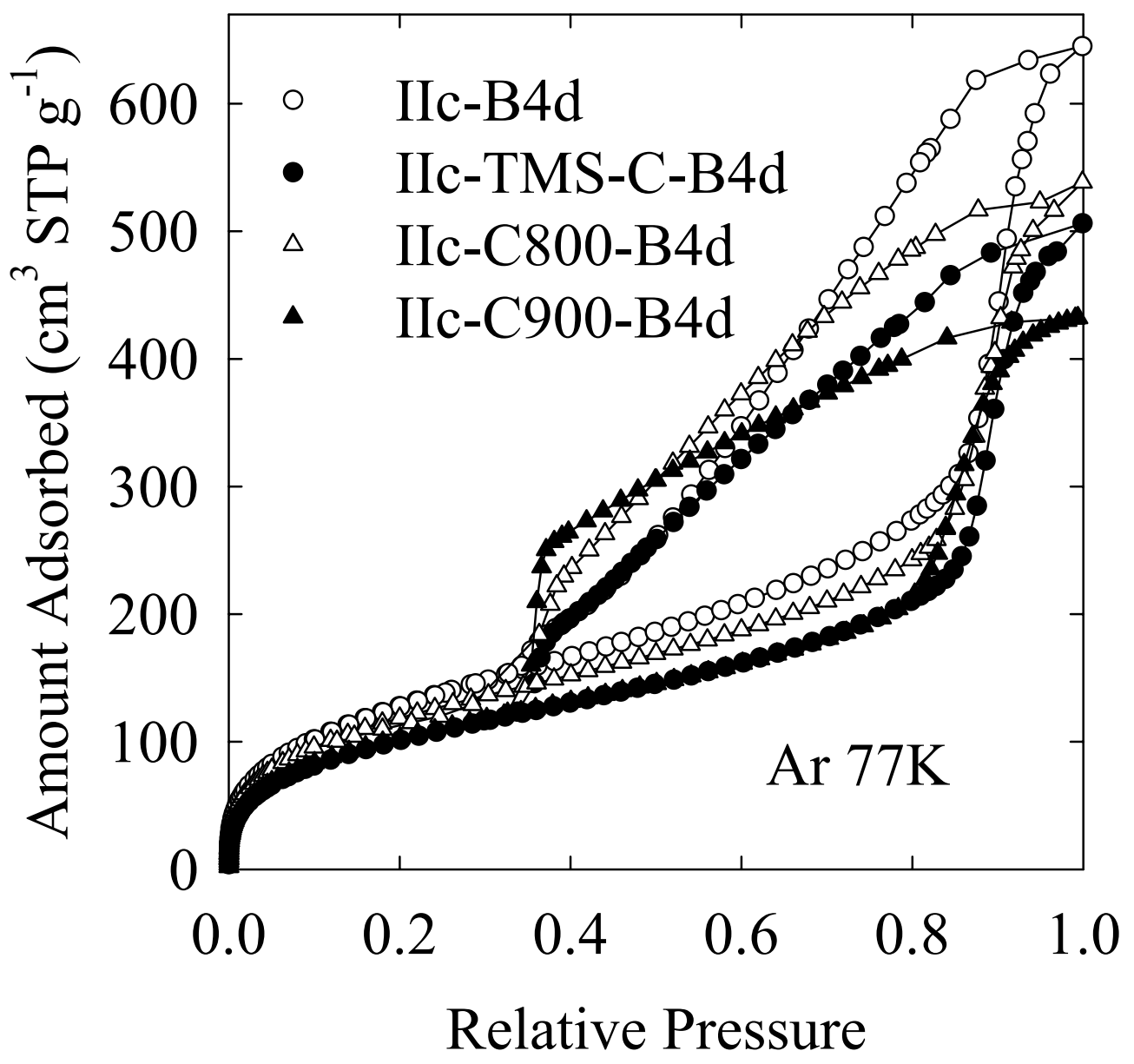

Supporting Figure 5S. Argon adsorption isotherms for FDU-1 samples (see caption to Figure 5) after boiling for 4 days. 
(c) American Chemical Society, Chem. Mater., Kruk et al., cm034911u Supporting Info Page S8

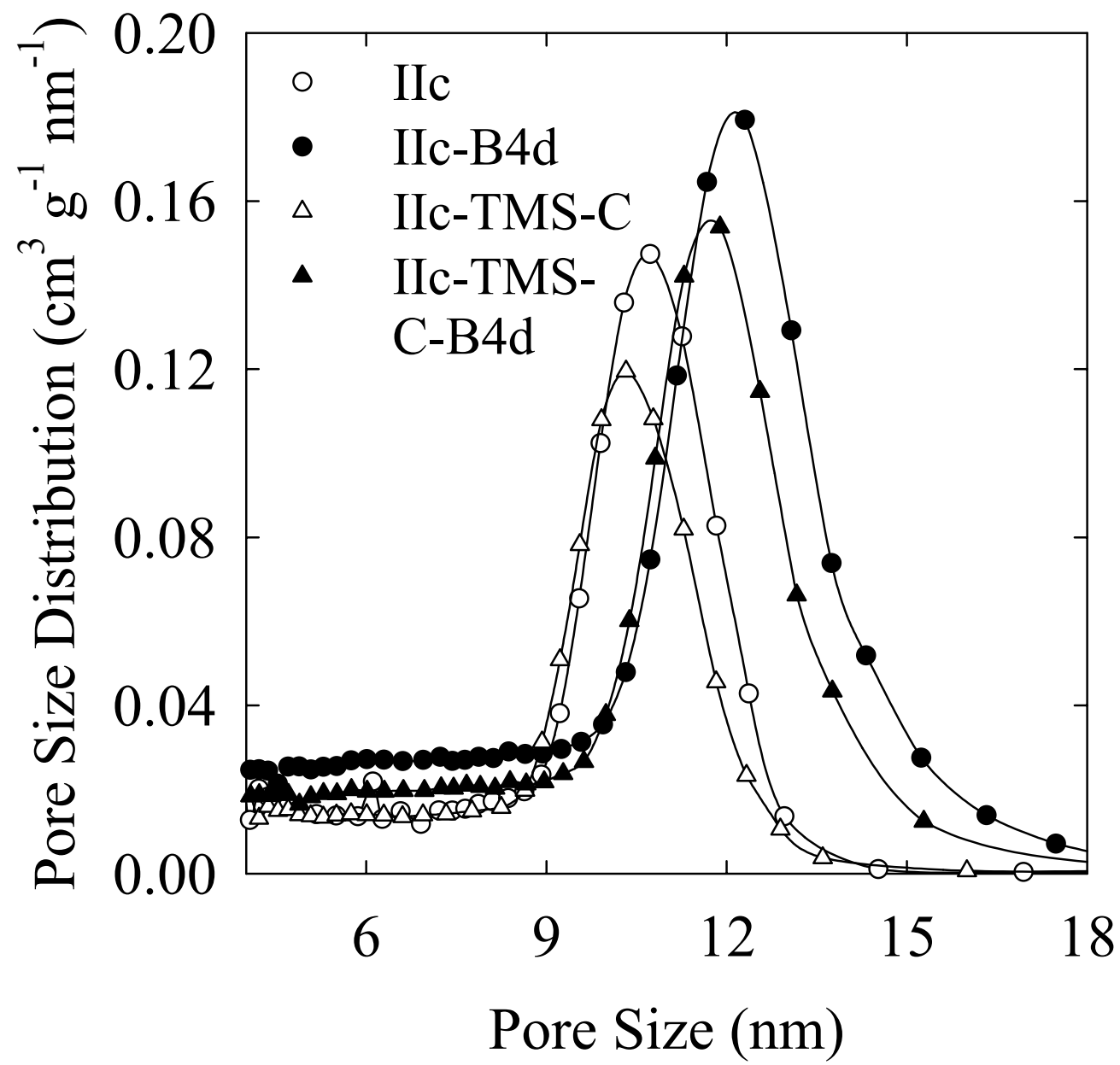

Supporting Figure 6S. Pore size distributions calculated from nitrogen adsorption isotherms for FDU-1 sample IIc (i) calcined at $813 \mathrm{~K}$ and (ii) calcined at $813 \mathrm{~K}$, modified with TMS and calcined at $813 \mathrm{~K}$, before and after boiling for 4 days. 
(c) American Chemical Society, Chem. Mater., Kruk et al., cm034911u Supporting Info Page S9

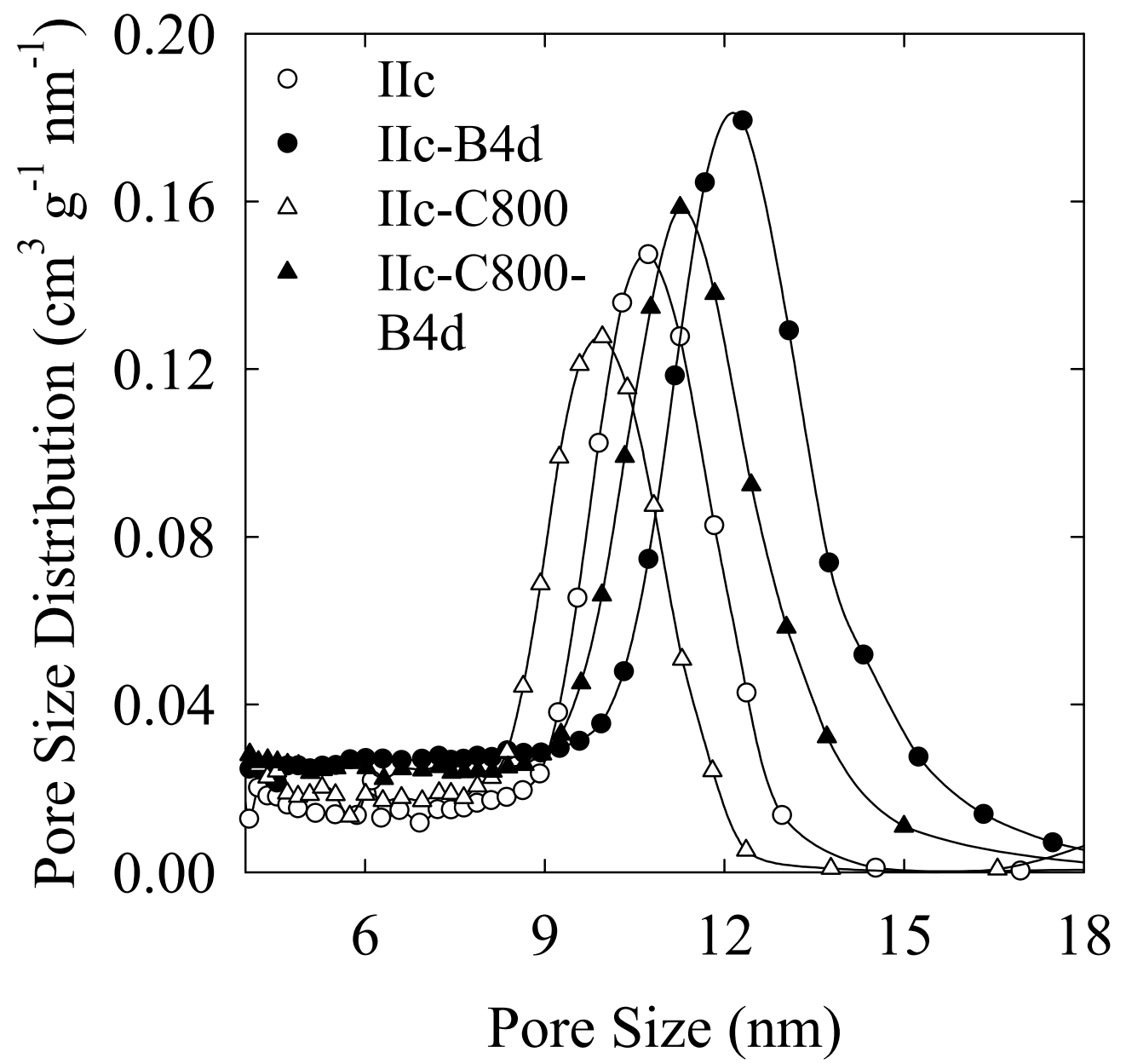

Supporting Figure 7S. Pore size distributions calculated from nitrogen adsorption isotherms for FDU-1 sample IIc calcined at $813 \mathrm{~K}$ and $1073 \mathrm{~K}$, before and after boiling for 4 days. 
(C) American Chemical Society, Chem. Mater., Kruk et al., cm034911u Supporting Info Page S10

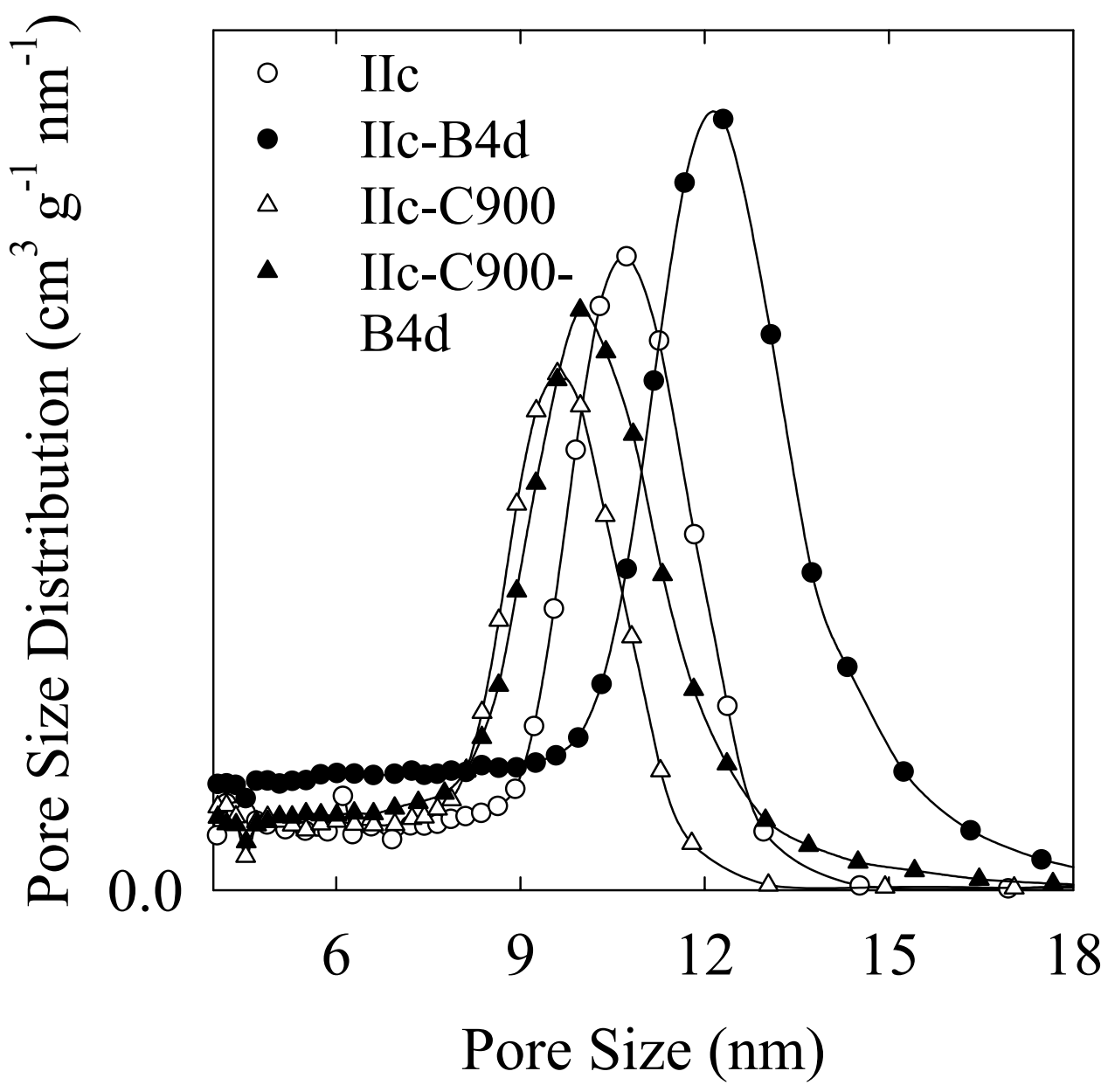

Supporting Figure 8S. Pore size distributions calculated from nitrogen adsorption isotherms for FDU-1 sample IIc calcined at $813 \mathrm{~K}$ and $1173 \mathrm{~K}$, before and after boiling for 4 days. 
(๖ American Chemical Society, Chem. Mater., Kruk et al., cm034911u Supporting Info Page S11

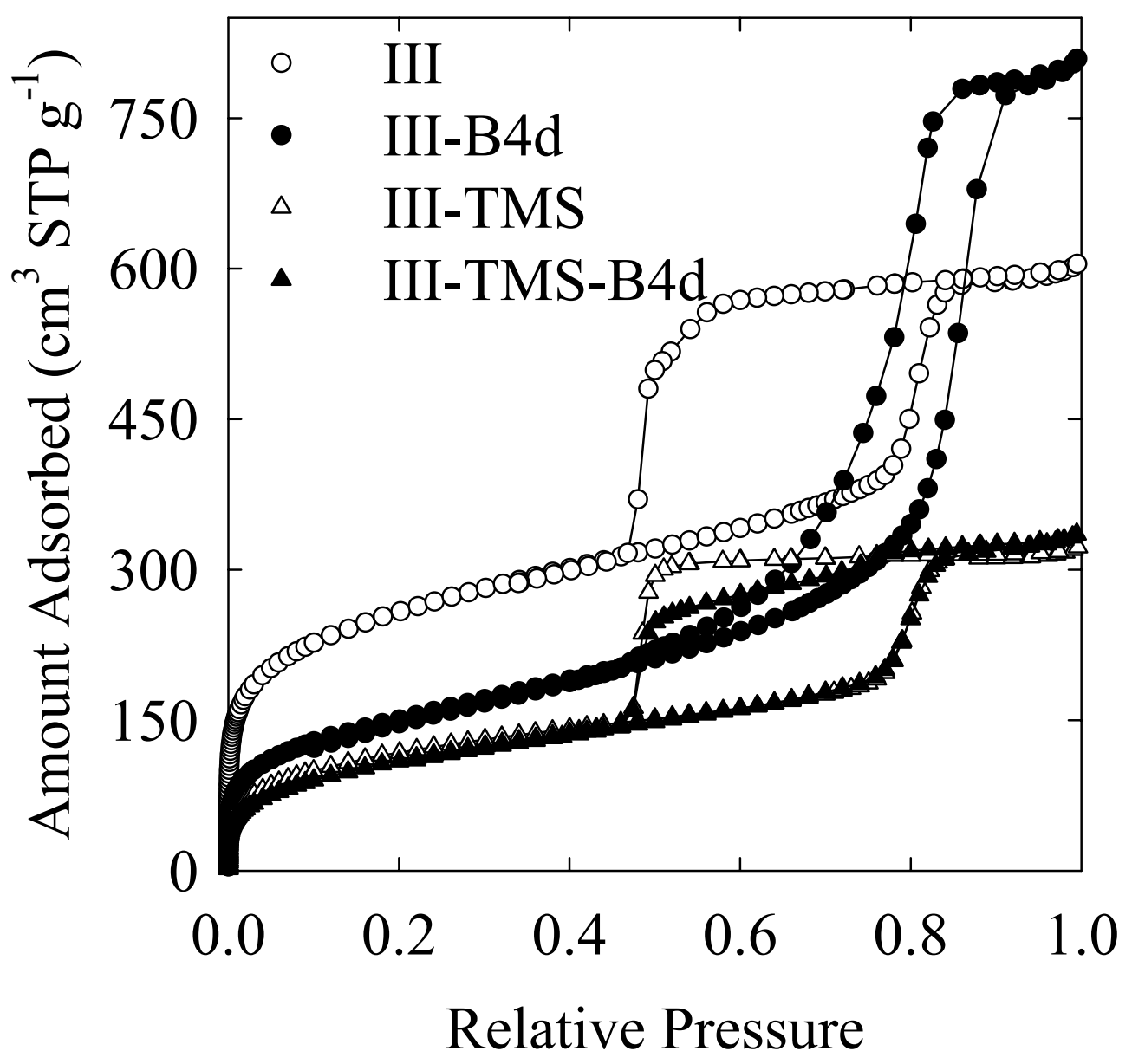

Supporting Figure 9S. Nitrogen adsorption isotherms for FDU-1 sample III: (i) calcined at 813 $\mathrm{K}$; (ii) calcined at $813 \mathrm{~K}$ and modified with TMS, both before and after boiling for 4 days. 
(c) American Chemical Society, Chem. Mater., Kruk et al., cm034911u Supporting Info Page S12

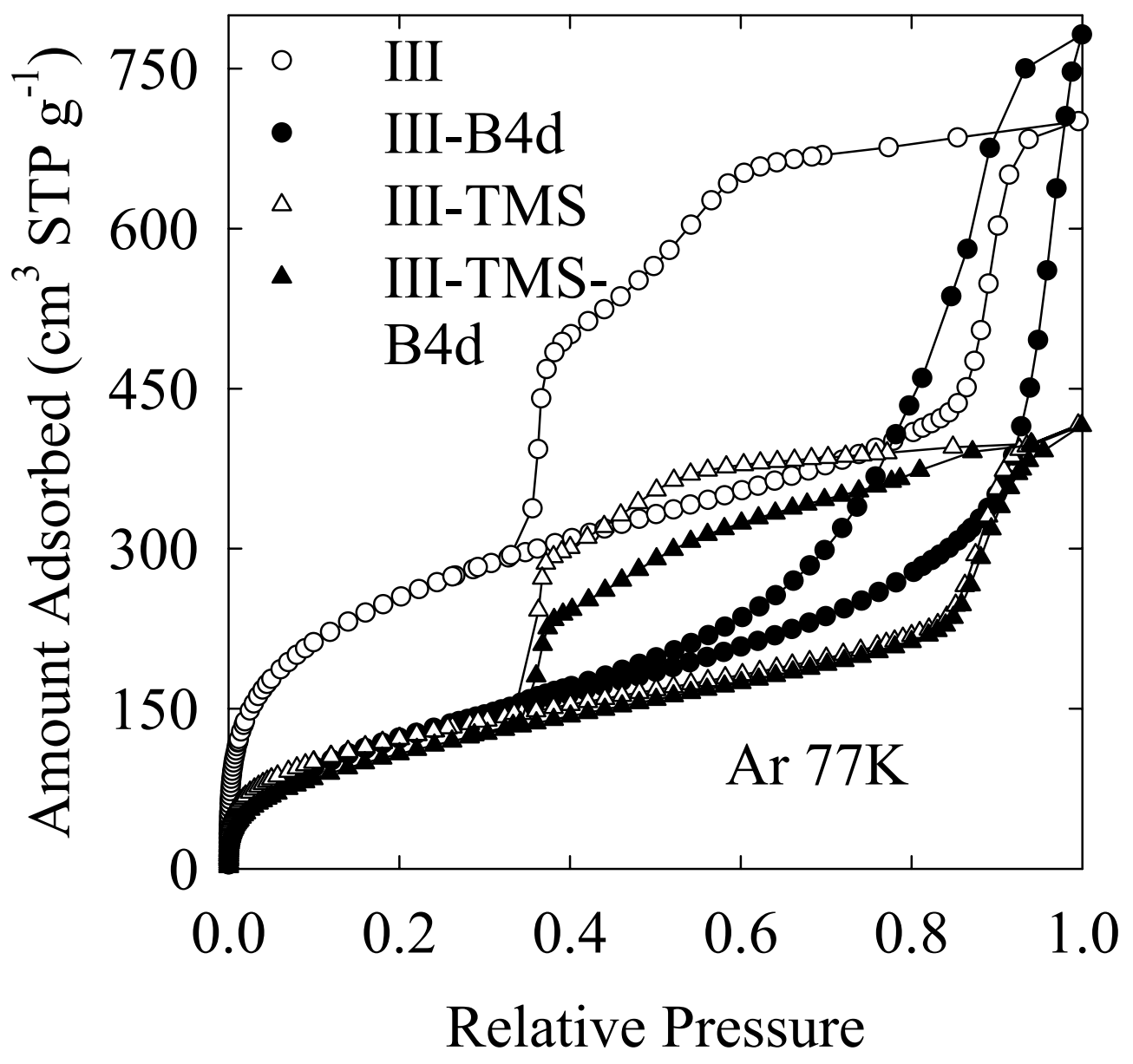

Supporting Figure 10S. Argon adsorption isotherms for FDU-1 sample III: (i) calcined at 813 $\mathrm{K}$; (ii) calcined at $813 \mathrm{~K}$ and modified with TMS, both before and after boiling for 4 days. 
(C) American Chemical Society, Chem. Mater., Kruk et al., cm034911u Supporting Info Page S13

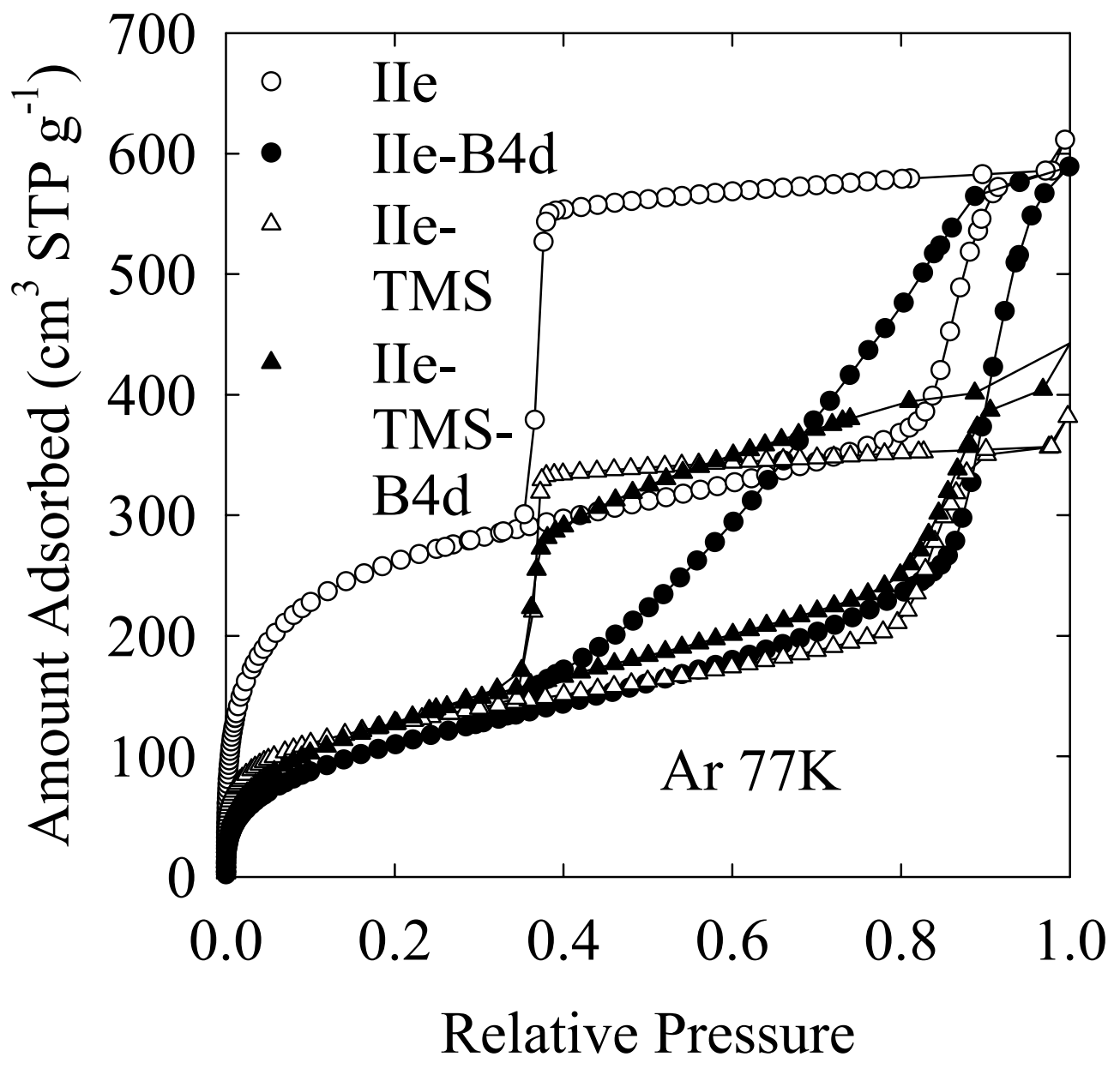

Supporting Figure 11S. Argon adsorption isotherms for FDU-1 sample IIe: (i) calcined at 813 $\mathrm{K}$; (ii) calcined at $813 \mathrm{~K}$ and modified with TMS, both before and after boiling for 4 days. 
(c) American Chemical Society, Chem. Mater., Kruk et al., cm034911u Supporting Info Page S14

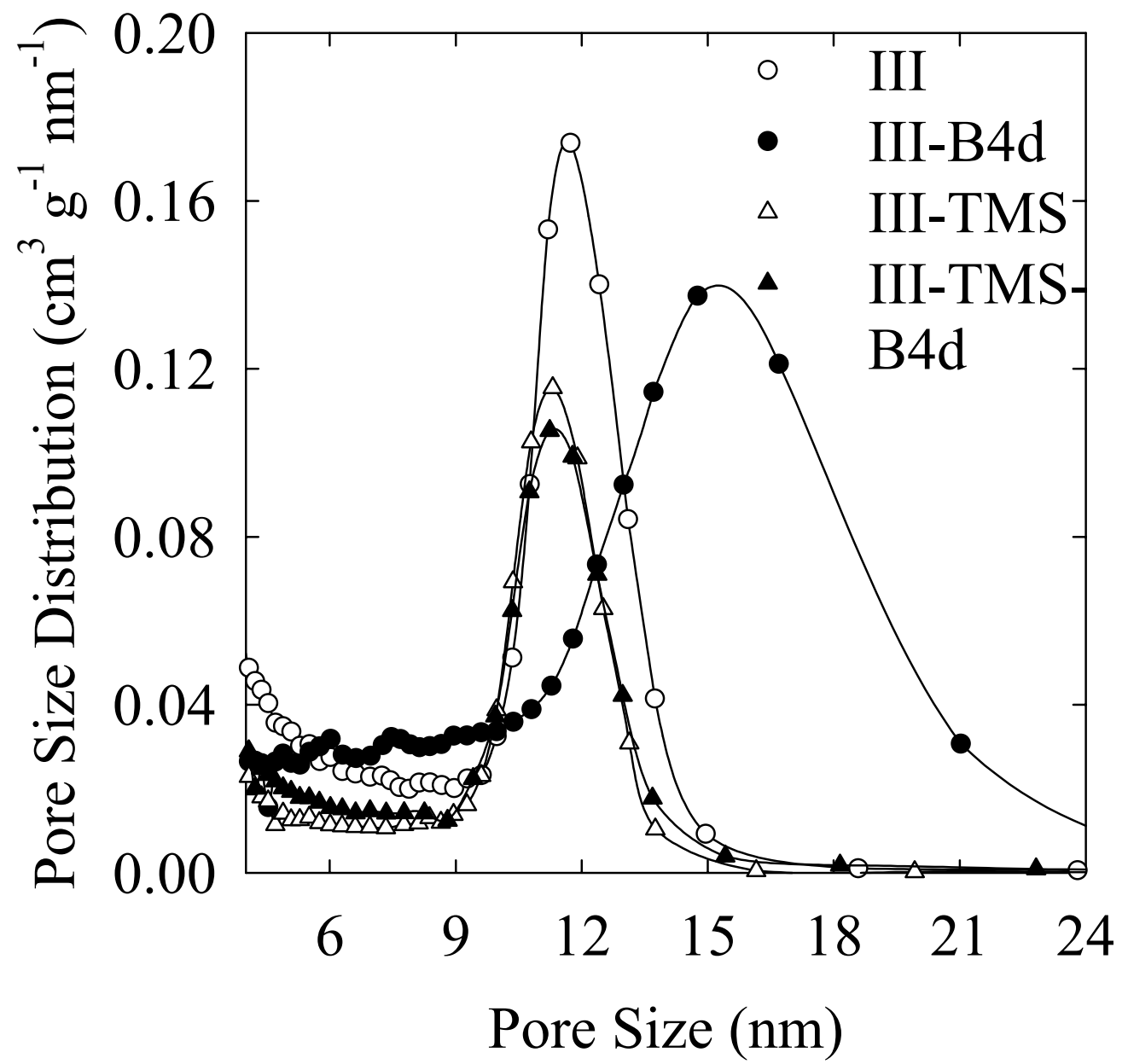

Supporting Figure 12S. Pore size distributions calculated from nitrogen adsorption isotherms for FDU-1 sample III: (i) calcined at $813 \mathrm{~K}$; (ii) calcined at $813 \mathrm{~K}$ and modified with TMS, before and after boiling for 4 days. 
(c) American Chemical Society, Chem. Mater., Kruk et al., cm034911u Supporting Info Page S15

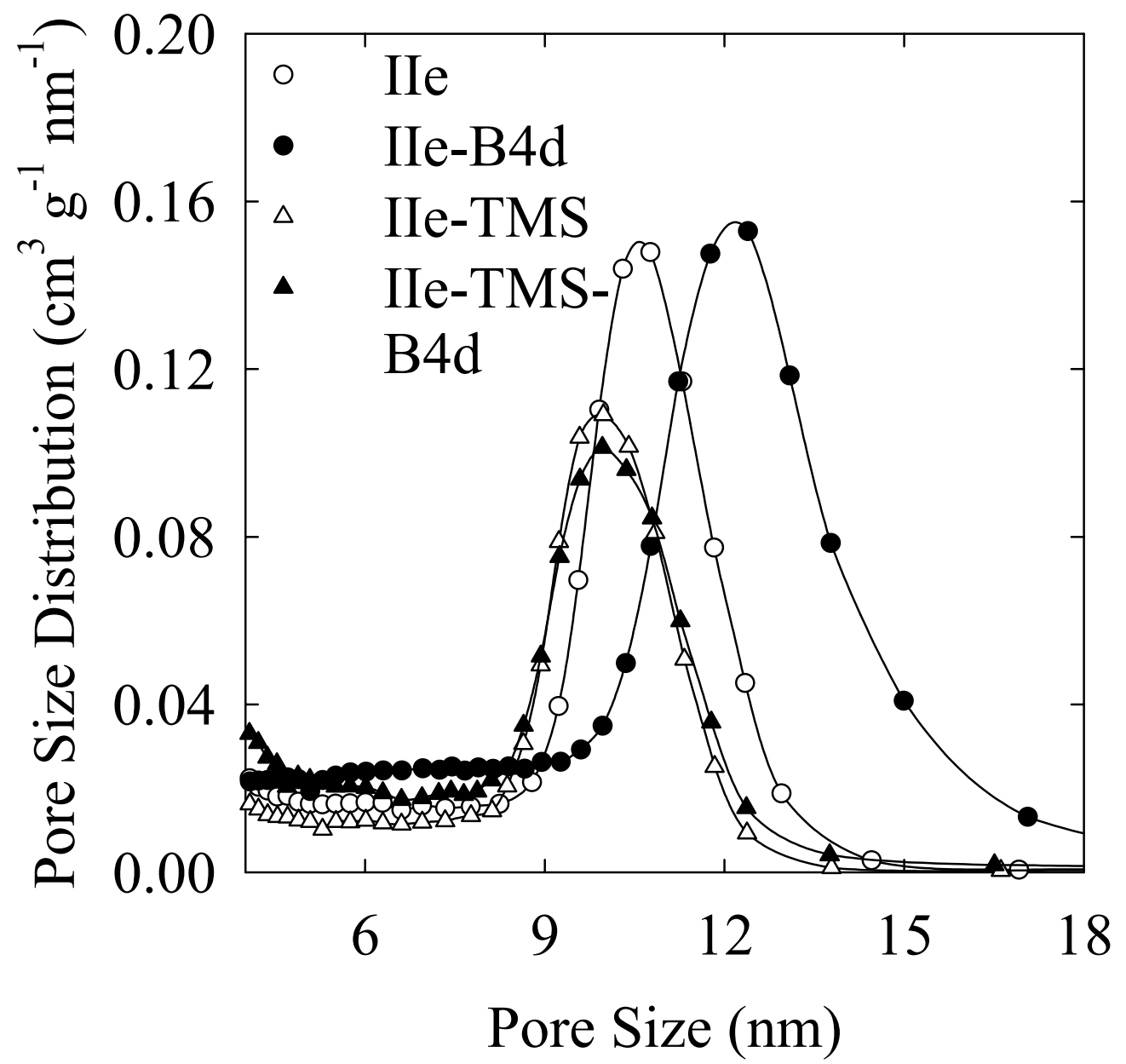

Supporting Figure 13S. Pore size distributions calculated from nitrogen adsorption isotherms for FDU-1 sample IIe: (i) calcined at $813 \mathrm{~K}$; (ii) calcined at $813 \mathrm{~K}$ and modified with TMS, before and after boiling for 4 days. 